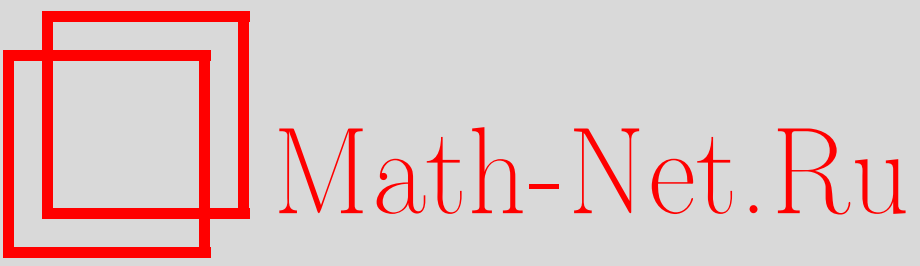

А. Ш. Шалданбаев, М. И. Акылбаев, М. Т. Шоманбаева, А. А. Шалданбаева, Критерий вольтерровости задачи Коши для уравнения пантографа, Итоги науки и техн. Сер. Соврем. мат. и ее прил. Темат. обз., 2019, том 171, 140-145

DOI: https://doi.org/10.36535/0233-6723-2019-171-140-145

Использование Общероссийского математического портала Math-Net.Ru подразумевает, что вы прочитали и согласны с пользовательским соглашением

http://www.mathnet.ru/rus/agreement

Параметры загрузки:

IP: 3.85 .5 .30

26 апреля 2023 г., 18:09:32 


\title{
КРИТЕРИЙ ВОЛЬТЕРРОВОСТИ ЗАДАЧИ КОШИ ДЛЯ УРАВНЕНИЯ ПАНТОГРАФА
}

\author{
(c) 2019 г. А. Ш. ШАЛДАНБАЕВ, М. И. АКЫЛБАЕВ, \\ М. Т. ШОМАНБАЕВА, А. А. ШАЛДАНБАЕВА
}

\begin{abstract}
АннотАция. В работе исследованы спектральные свойства задачи Коши уравнения пантографа и установлена граница изменения параметра, на которой задача остается вольтерровой.
\end{abstract}

Ключевые слова: уравнение пантографа, спектр, вполне непрерывность, ядерность, оператор Гильберта-Шмидта, формула следа оператора, формула Гаала.

\section{CRITERION FOR THE VOLTERRA PROPERTY OF THE CAUCHY PROBLEM FOR THE PANTOGRAPH EQUATION}

\author{
(C) 2019 A. SH. SHALDANBAYEV, M. I. AKYLBAYEV, \\ M. T. SHOMANBAYEVA, A. A. SHALDANBAYEVA
}

\begin{abstract}
In this paper, spectral properties of the Cauchy problem of the pantograph equation are examined and the boundary of the parameter range is established at which the problem remains a Volterra problem.

Keywords and phrases: pantograph equation, spectrum, complete continuity, nuclearity, HilbertSchmidt operator, operator trace formula, Gaal formula.
\end{abstract}

AMS Subject Classification: 34K06, 34K08

1. Введение. Уравнения типа пантографа с начала 1970 гг. изучались в работах Т. Като [7], А. Изерлеса [6] и других авторов. Такие уравнения возникают в самых разных областях: астрофизике (В. А. Амбарцумян, поглощение света межзвездной материей), технике (Дж. Окендон, А. Б. Тайлер, математическая модель контактного провода электроснабжения подвижного состава), биологии (А. Дж. Холл, Г. С. Уэйк, моделирование процесса роста и деление клеток). Рассматривались вопросы разрешимости начальной задачи и различных ее обобщений, асимптотического поведения решений на бесконечности, существования периодических и почти периодических решений, в основном для уравнения первого порядка (уравнение пантографа $\left.y^{\prime}=a y(\lambda t)+b y(t)\right)$.

Рассмотрим в пространстве $L^{2}(0,1)$ задачу Коши

$$
y^{\prime}(x)=\lambda y(\alpha x), \quad 0 \leqslant x \leqslant 1, \quad y(0)=0 .
$$

Если $\lambda=0$, то $y^{\prime}(x)=0, y(x)=$ const $=y(0)=0$, так что $y(x) \equiv 0$, поэтому существует обратный оператор. Проинтегрировав обе части уравнения (1), имеем

$$
y(x)=\lambda \int_{0}^{x} y(\alpha t) d t, \quad \int_{0}^{x} y(\alpha t) d t=\frac{y(x)}{\lambda} ;
$$


следовательно, обратный оператор имеет вид

$$
A y(x)=\int_{0}^{x} y(\alpha t) d t, \quad 0 \leqslant x \leqslant 1 .
$$

Выясним, при каких значениях $\alpha$ оператор $A$ вольтерров, т.е. не имеет собственных значений, отличных от нуля.

2. Метод исследования. Будем опираться на спектральную теорию операторов ГильбертаШмидта. Напомним, что вполне непрерывный оператор $T$, действующий в гильбертовом пространстве $\mathfrak{H}$, называется оператором типа Гильберта-Шмидта, если

$$
\sum_{n=1}^{\infty}\left(T \varphi_{n}, T \varphi_{n}\right)<\infty
$$

где $\varphi_{n}, n=1,2, \ldots$, - некоторый ортонормированный базис в $\mathfrak{H}$. Круглыми скобками здесь и в дальнейшем обозначается скалярное произведение.

В случае интегрального оператора

$$
K f=\int_{a}^{b} k(x, t) f(t) d t,
$$

действующего в $L^{2}(a, b)$, условие (3) может быть записано в виде

$$
\int_{a}^{b} \int_{a}^{b}|k(x, t)|^{2} d x d t<\infty .
$$

В данной работе мы исследуем условия, при которых оператор $A$ оказывается вольтерровым.

Будем говорить, что вполне непрерывный оператор $P$ имеет след, если в любом ортонормированном базисе $\varphi_{i}, i=1,2, \ldots$, абсолютно сходится ряд

$$
\sum_{i=1}^{\infty}\left(P \varphi_{i}, \varphi_{i}\right)
$$

Заметим, что если ряд (4)сходится абсолютно в любом ортонормированном базисе, то сумма получается при этом всегда одна и та же; этот факт хорошо известен в случае самосопряженных операторов (см. [1]). Отметим, что абсолютную сходимость достаточно проверить в базисе, состоящем из собственных векторов.

В общем случае указанное утверждение может быть легко доказано разложением оператора в сумму вещественной и мнимой компоненты: $P=P_{R}+i P_{I}$, где $P_{R}$ и $P_{I}$ - самосопряженные операторы:

$$
P_{R}=\frac{1}{2}\left(P+P^{*}\right), \quad P_{I}=\frac{1}{2 i}\left(P-P^{*}\right) .
$$

Действительно, вместе с рядом (4) абсолютно сходятся ряды

$$
\sum_{i=1}^{\infty}\left(P_{R} \varphi_{i}, \varphi_{i}\right), \quad \sum_{i=1}^{\infty}\left(P_{I} \varphi_{i}, \varphi_{i}\right),
$$

ибо $\left(P_{R} \varphi_{i}, \varphi_{i}\right)=\operatorname{Re}\left(P \varphi_{i}, \varphi_{i}\right)$ и $\left(P_{I} \varphi_{i}, \varphi_{i}\right)=\operatorname{Im}\left(P \varphi_{i}, \varphi_{i}\right)$. Так как суммы последних двух рядов не зависят от выбора базиса, то этим свойством обладает, очевидно, и сумма (4). След оператора $P$ будем обозначать $\operatorname{sp} P$.

Очевидно, если $P$ - самосопряженный оператор, имеющий след, то

$$
\text { sp } P=\sum_{i=1}^{\infty} \mu_{i}
$$

где $\mu_{i}$ - собственные значения оператора $P$. 
Лемма 1. Если $T_{1}$ и $T_{2}$-операторы типа Гильберта-Шмидта, то оператор $T_{1} T_{2}$ имеет след.

Доказательство. Действительно,

$$
\left|\left(T_{1} T_{2} \varphi_{i}, \varphi_{i}\right)\right| \leqslant\left\|T_{2} \varphi_{i}\right\| \cdot\left\|T_{1}^{*} \varphi_{i}\right\| \leqslant \frac{1}{2}\left(\left\|T_{2} \varphi_{i}\right\|^{2}+\left\|T_{1}^{*} \varphi_{i}\right\|^{2}\right)
$$

Сходимость ряда, составленного из правых частей последнего неравенства, в любом ортонормированном базисе доказывает лемму.

Следствие 1. Если T-оператор типа Гильберта-Шмидта, то при $n \geqslant 2$ оператор $T^{n}$ имеет след.

Остановимся несколько подробнее на интегральных операторах, действующих в $L^{2}(0,1)$. Рассмотрим интегральный оператор $K$ типа Гильберта-Шмидта

$$
K f=\int_{0}^{1} K(x, t) f(t) d t
$$

Как уже указывалось, все степени оператора $K$, начиная со второй, имеют след.

Лемма 2. Следы интегральных операторов $K^{n}, n=2,3, \ldots$, могут быть вычислены по формуле

$$
\operatorname{sp} K^{n}=\int_{0}^{1} \ldots \int_{0}^{1} K\left(x_{1}, x_{2}\right) K\left(x_{2}, x_{3}\right) \ldots K\left(x_{n}, x_{1}\right) d x_{1} d x_{2} \ldots d x_{n} .
$$

Указанный факт хорошо известен в случае эрмитовых ядер. Его доказательство в общем случае мы сведем к следующему предложению: пусть $K_{1}$ и $K_{2}$-самосопряженные интегральные операторы типа Гильберта-Шмидта. Обозначим через $K_{1}\left(x_{1}, x_{2}\right), K_{2}\left(x_{1}, x_{2}\right)$ их ядра. Тогда справедливо равенство

$$
\operatorname{sp} K_{1} K_{2}=\int_{0}^{1} \int_{0}^{1} K_{1}\left(x_{1}, x_{2}\right) K_{2}\left(x_{2}, x_{1}\right) d x_{1} d x_{2} .
$$

Доказательство. Обозначим правую часть предполагаемого равенства через $I$ и представим ее в виде

$$
I=\frac{1}{4}\left(\int_{0}^{1} \int_{0}^{1}\left|K_{1}+K_{2}\right|^{2} d x_{1} d x_{2}-\int_{0}^{1} \int_{0}^{1}\left|K_{1}-K_{2}\right|^{2} d x_{1} d x_{2}\right) .
$$

Равенство (10) легко проверяется. Следует лишь учесть, что ядра $K_{1}\left(x_{1}, x_{2}\right)$ и $K_{2}\left(x_{1}, x_{2}\right)$ эрмитовы: $K\left(x_{1}, x_{2}\right)=\overline{K\left(x_{2}, x_{1}\right)}$.

Заметим теперь (см. [4]), что первый из интегралов справа в формуле (10) равен сумме квадратов собственных значений интегрального оператора $K_{1}+K_{2}$; последняя в силу (6) совпадает со следом оператора $\left(K_{1}+K_{2}\right)^{2}$. Итак,

$$
\int_{0}^{1} \int_{0}^{1}\left|K_{1}+K_{2}\right|^{2} d x_{1} d x_{2}=\operatorname{sp}\left(K_{1}+K_{2}\right)^{2}
$$

Аналогично находим

$$
\int_{0}^{1} \int_{0}^{1}\left|K_{1}-K_{2}\right|^{2} d x_{1} d x_{2}=\operatorname{sp}\left(K_{1}-K_{2}\right)^{2}
$$

В результате приходим к равенству

$$
I=\frac{1}{4}\left(\operatorname{sp}\left(K_{1}+K_{2}\right)^{2}-\operatorname{sp}\left(K_{1}-K_{2}\right)^{2}\right)
$$


Раскрывая скобки и учитывая формулу sp $K_{1} K_{2}=\operatorname{sp} K_{2} K_{1}$, приходим к формуле (9).

Для доказательства леммы запишем оператор $K^{n}$ в виде

$$
K^{n}=K \cdot K^{n-1}
$$

и каждый из операторов $K$ и $K^{n-1}$ разложим в сумму вещественной и мнимой компоненты:

$$
K=K_{R}+i K_{I}, \quad K^{n-1}=\left(K^{n-1}\right)_{R}+i\left(K^{n-1}\right)_{I} .
$$

Внеся эти разложения в правую часть (11) и раскрыв скобки, получим линейную комбинацию операторов вида $K_{1} \cdot K_{2}$. Проделав аналогичные преобразования с ядром $K^{n}$ и воспользовавшись затем равенством (9), приходим к заключению леммы.

Сделаем в заключение одно замечание в связи с формулой (8). Обозначим через $K^{n}(x, t)$ ядро оператора $K^{n}$, определяемое формулой

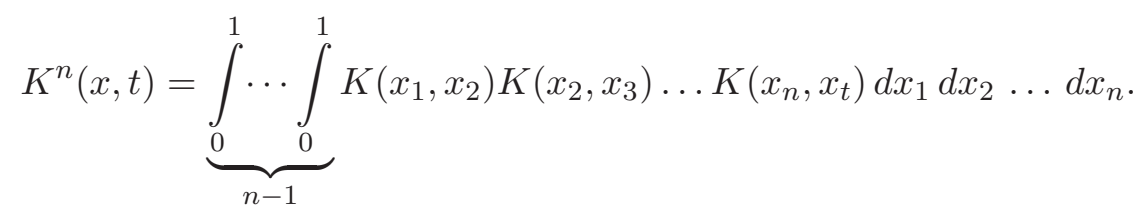

Пользуясь теоремой Фубини, равенство (8) можно, очевидно, записать в виде

$$
\operatorname{sp} K^{n}=\int_{0}^{1} K^{(n)}(t, t) d t .
$$

Следствие 2. При $n=2$ формула (12) принимает вид

$$
\operatorname{sp} K^{2}=\int_{0}^{1} K^{(2)}(t, t) d t, \quad \text { əде } \quad K^{(2)}(x, t)=\int_{0}^{1} K(x, s) K(s, t) d s .
$$

Эти формулы составляют основу нашего метода. Заметим, что многомерный вариант формулы (13) называется формулой Гаала (см. [5]).

\section{3. Результаты исследований.}

\section{Теорема 1.}

(a) Если $0<\alpha \leqslant 1, \operatorname{mo~sp~} A^{2}=0$.

(b) Если $\alpha>1$, mo

$$
\operatorname{sp} A^{2}=\frac{1}{\alpha^{2}}\left(1-\frac{1}{\alpha}\right)
$$

Доказательство. Оператор $A$ имеет вид

$$
A y(x)=\int_{0}^{x} y(\alpha t) d t, \quad 0 \leqslant x \leqslant 1 .
$$

Полагая $\xi=\alpha t$, сделаем замену переменной в этом интеграле:

$$
\int_{0}^{x} y(\alpha t) d t=\left\{\begin{array}{l}
\alpha t=\xi \\
t=\xi / \alpha, \\
d t=d \xi / \alpha
\end{array}\right\}=\int_{0}^{\alpha x} \frac{y(\xi) d \xi}{\alpha}=\int_{0}^{1} \frac{\theta(\alpha x-\xi)}{\alpha} y(\xi) d \xi=\int_{0}^{1} K(x, \xi) y(\xi) d \xi
$$

где

$$
K(x, \xi)=\frac{\theta(a x-\xi)}{\alpha}, \quad \theta(x)= \begin{cases}1, & \text { если } x \geqslant 0 \\ 0, & \text { если } x<0\end{cases}
$$


Ядро $K(x, \xi)$ ограничено, поэтому $K$ является оператором типа Гильберта-Шмидта, а его квадрат $K^{2}$-ядерным оператором. Вычислим ядро оператора $K^{2}$ :

$$
K^{2}(x, \xi)=\int_{0}^{1} K(x, \eta) K(\eta, \xi) d \eta=\frac{1}{\alpha^{2}} \int_{0}^{1} \theta(\alpha x-\eta) \theta(\alpha \eta-\xi) d \eta .
$$

Следовательно,

$$
K^{2}(x, x)=\frac{1}{\alpha^{2}} \int_{0}^{1} \theta(\alpha x-\eta) \theta(\alpha \eta-x) d \eta
$$

По формуле Гаала имеем

$$
\operatorname{sp} A^{2}=\int_{0}^{1} K^{2}(x, x) d x=\frac{1}{\alpha^{2}} \int_{0}^{1} \int_{0}^{1} \theta(\alpha x-\eta) \theta(\alpha \eta-x) d \eta d x .
$$

Полагая

$$
\Phi(x, \eta)=\frac{\theta(\alpha x-\eta) \theta(\alpha \eta-x)}{\alpha^{2}},
$$

имеем

$$
\Phi(\eta, x)=\frac{\theta(\alpha \eta-x) \theta(\alpha x-\eta)}{\alpha^{2}}=\Phi(x, \eta),
$$

поэтому

$$
\begin{aligned}
\operatorname{sp} A^{2}=2 \int_{0}^{1} d x \int_{0}^{x} \frac{\theta(\alpha x-\eta) \theta(\alpha \eta-x) d \eta}{\alpha^{2}}=\frac{2}{\alpha^{2}} \int_{0}^{1} d x \int_{x / \alpha}^{x} d \eta & =\frac{2}{\alpha^{2}} \int_{0}^{1}\left(x-\frac{x}{\alpha}\right) d x= \\
& =\frac{2}{\alpha^{2}}\left(1-\frac{1}{\alpha}\right) \int_{0}^{1} x d x=\frac{1}{\alpha^{2}}\left(1-\frac{1}{\alpha}\right) .
\end{aligned}
$$

Если $0<\alpha \leqslant 1$, то $\alpha x \leqslant x$, откуда последовательно заключаем, что $0<\eta \leqslant \alpha x \leqslant x, \alpha \eta \leqslant \eta \leqslant x$, $\theta(\alpha \eta-x)=0$ и $\operatorname{sp} A^{2}=0$.

Теорема 2. Оператор А вольтерров тогда и только тогда, когда $0<\alpha \leqslant 1$.

Доказательство. Необходимость. Если оператор $A$ вольтерров, то оператор $A^{2}$ также вольтерров. Тогда по теореме Лидского (см. [2]) имеем $\operatorname{sp} A^{2}=0$. Если $\alpha>1$, то $\operatorname{sp} A^{2}>0$, поэтому $0<\alpha \leqslant 1$.

Достаточность следует из теоремы Нерсесяна (см. [3]).

4. Обсуждение. В случае $0<\alpha<1$ аргумент уравнения $y^{\prime}(x)=\lambda y(\alpha x)$ является запаздывающим, а в случае $\alpha>1$-опережающим. По-видимому, в случае запаздывания аргумента задача Коши всегда вольтеррова, а в случае опережающего аргумента появляется спектр, иначе говоря, задача разрешима не для всех значений параметра $\lambda$. В точках спектра нарушается единственность, т.е. задача некорректна.

Замечание. При решении краевой задачи для обыкновенного дифференциального уравнения первого порядка обычно подразумевается, что аргумент искомой функции лежит в заданном сегменте, но, как известно, области определения найденных собственных функций шире, чем первоначальная область краевой задачи. Мы полагаем, что в нашей задаче также имеет место подобный случай. 


\section{СПИСОК ЛИТЕРАТУРЫ}

1. Гохберг Н. Ц., Крейн М. Г. Основные положения о дефектных числах, корневых числах и индексах линейных операторов// Усп. мат. наук. - 1957. - 12, № 2 (74).

2. Лидский В. Б. Несамосопряженные операторы, имеющие след// Докл. АН СССР. - 1959. - 125, № 3. - C. $485-488$.

3. Нерсесян А. Б. К теории интегральных уравнений типа Вольтерра// Докл. АН СССР. - 1964. - 155, № 5. - C. 1006-1009.

4. Рисс Ф., Секефальви-Надъ Б. Лекции по функциональному анализу. - М.: ИЛ, 1954.

5. Brislawn C. Kernels of trace class operators// Proc. Am. Math. Soc. - 1988. - 104, № 4 - P. 1181-1190.

6. Iserless A. On the generalized functional-differential equation// Eur. J. Appl. Math. — 1993. — 4. P. $1-38$.

7. Kato T., Mcleod J. B. Functional-differential equation $y^{\prime}=a y(\lambda t)+b y(t) / /$ Bull. Am. Math. Soc. -1971 . — 77, № 6. - P. 891-937.

Шалданбаев Амир Шалданбаевич

Международный университет SILKWAY, Шымкент, Казахстан

E-mail: shaldanbaev51@mail.ru

Акылбаев Мусабек Исламович

Региональный социально-инновационный университет, Шымкент, Казахстан

E-mail: musabek_kz@mail.ru

Шоманбаева Манат Торгаевна

Южно-Казахстанский государственный университет им. М. Ауэзова, Шымкент, Казахстан

E-mail: mtshomanbaeva@mail.ru

Шалданбаева Айгерим Амировна

Региональный социально-инновационный университет, Шымкент, Казахстан

E-mail: Altima_a@mail.ru 